\title{
Edge dislocation in a vertical smectic- $A$ film: Line tension versus film thickness and Burgers vector
}

\author{
Jean-Christophe Géminard, Claude Laroche, and Patrick Oswald \\ Laboratoire de Physique de l'Ecole Normale Supérieure de Lyon, 46 Allée d'Italie, 69364 Lyon Cedex, France
}

(Received 13 March 1998; revised manuscript received 22 July 1998)

\begin{abstract}
The equilibrium shape of a looped edge dislocation in a vertical smectic- $A$ film allows the determination of its line tension: in agreement with theoretical predictions, it is found to be proportional to its Burgers vector and to increase as $1 / \sqrt{d}$ when the film thickness $d$ decreases. [S1063-651X(98)06411-3]

PACS number(s): 61.30.Jf, 83.70.Jr, 68.15.+e, 51.20.+d
\end{abstract}

Focal conics [1] and edge dislocations [2] are the most common defects of the smectic- $A$ phase. The edge dislocations can group together to form giant dislocations, the socalled oily streaks of the lamellar phases. This property results from the special elasticity of the smectic- $A$ phase and is consistent with the theoretical prediction that the line energy $T$ of an edge dislocation in an infinite medium must be proportional to its Burgers vector $b$ [3] rather than to $b^{2}$ as in usual solids [4]. To our knowledge, there have not been any experimental direct measurements of the line energy as a function of the Burgers vector. The interaction of a dislocation with a free surface of surface tension $\gamma$ has also been studied theoretically [5]: Edge dislocations are repelled from the surface when $\gamma>\sqrt{K B}$ (where $K$ and $B$ are, respectively, the curvature and the compressibility moduli). As a consequence, dislocations must locate in the midplane of free standing films, which has been already observed experimentally [6,7], but a direct measurement of the interaction energy with the surfaces failed.

In this article we focus our attention on static properties of edge dislocations in free-standing films (Fig. 1). We have two objectives: an experimental measurement of the line tension $T$ of an edge dislocation first, as a function of the film thickness $D$, and, second, as a function of the Burgers vector $b$ when surface effects are negligible.

In practice, free-standing smectic films are obtained by spreading out a dropplet of the smectic- $A$ phase on a frame [8]. When the film is horizontal and in mechanical equilibrium with air, its thickness is homogeneous, except near the edges, where a meniscus forms [6,7]. The film is then in a metastable state and experiences a pressure difference $\Delta P$ $=P_{\text {air }}-P_{\text {smectic }}$ whose value is fixed by the meniscus, which acts as a reservoir. (This $\Delta P$ is equilibrated by a layer compression.) Experimentally, the meniscus profile is circular and its radius of curvature $R$ is related to $\Delta P$ via the Laplace equation $\Delta P=\gamma / R$. Recently, we showed how to measure the pressure difference across the film surfaces by determining the meniscus profile at the edges [9]. We also showed how to nucleate elementary edge-dislocation loops by locally heating the film, and we measured their critical radius of nucleation $R_{c}$. From these two quantities, $R_{c}$ and $\Delta P$, we could calculate the line tension $T$ of a dislocation according to the relation $R_{c}=T / b \Delta P$.

Since the critical radius of nucleation $R_{c}$ is difficult to measure, we developed a method for measuring the line ten- sion of dislocations. It is inspired by the sessile drop method [10] used to measure the surface tension of usual liquids and consists of observing the flattening of a drop deposited on a planar solid surface when the drop size is large enough (i.e., about the capillary length $\sqrt{\gamma / \rho g}$ where $\gamma, \rho$, and $g$ are respectively the surface tension, the density and the gravity constant). The same holds for a dislocation in a vertical film provided its size is about $\sqrt{T / \rho g}$ (i.e., a few tens of micrometers).

We now decribe theoretically the equilibrium state of a vertical smectic film. The pressure $P_{\text {smectic }}$ depends on the height $z$ measured from the bottom edge of the frame. Because the smectic layers are fluid, the local pressure difference $\Delta P$ is

$$
\Delta P(z)=P_{\text {air }}-\left(P_{0}-\rho g z\right)=\Delta P_{0}+\rho g z,
$$

where $\rho$ is the density of the liquid crystal, $g$ the gravity constant, and $P_{0}$ the pressure inside the film at $z=0$. We now nucleate a dislocation loop. According to Archimedes' law, the loop (which is similar to a bubble) goes up and quickly reaches the top edge of the frame where it stabilizes while deforming under the action of gravity. A few minutes later, hydrodynamic currents are again negligible and the surface area of the loop no longer changes significantly. The loop equilibrium shape satisfies the following equation:

$$
T \kappa=b \Delta P(z),
$$

where $\kappa$ is the local curvature of the dislocation. This equation can be solved numerically in polar coordinates by using

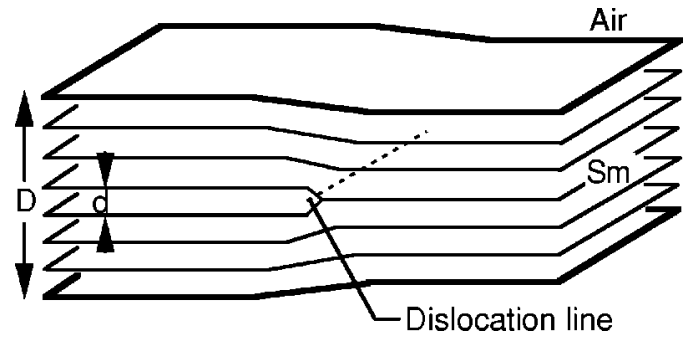

FIG. 1. Edge dislocation in a smectic- $A$ film: An edgedislocation line is defined to be the defect line that bounds two domains of the film that have different thicknesses $D$. The Burgers vector $b$ is then defined to be the thickness difference ( $b$ is an integer multiple of the layer thickness $d$ ). 


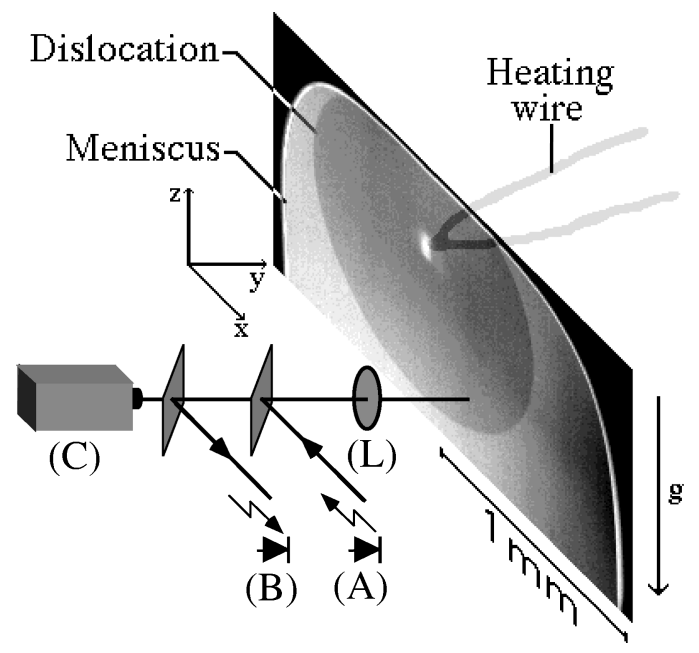

FIG. 2. Experimental setup: The vertical smectic film is observed via reflecting microscopy in monochromatic light. The objective lens $(L)$ casts the image of the film on the CCD camera $(C)$. The light source is the red LED $(A)$. The photodiode $(B)$ measures the intensity of the reflected light. The heating wire, used to nucleate the dislocation loops, is placed about $50 \mu \mathrm{m}$ behind the film. (The loop shown in the picture is an experimental one.)

the classical Runge-Kutta method [11]. Fitting the experimental shape to the theoretical one allows us to deduce $T$ and $\Delta P_{0}$. The free parameters are the position $(x, z)$ of the lower point of the loop, $\Delta P_{0}$, and the line tension $T$ (which is experimentally determined within $15 \%$ ).

The experiments are performed with the liquid crystal $8 \mathrm{CB}$ (4'-n-octyl-4-cyanobiphenyl) at room temperature (about $21{ }^{\circ} \mathrm{C}$; this compound has a smectic- $A$ phase between $20^{\circ} \mathrm{C}$ and $33.4{ }^{\circ} \mathrm{C}$ ). The smectic film is streched on a rigid rectangular frame $(2 \mathrm{~mm}$ wide, $4 \mathrm{~mm}$ high, and $0.5 \mathrm{~mm}$ thick). After about one hour the film is stable and perfectly homogeneous without visible flow. The film is observed through an objective lens, which casts the image on a CCD (charge-coupled-device) camera (Fig. 2). The camera, the lens, the light source, and a beam splitter are placed on a T-shaped optical bench and form a common reflection microscope. The frame is mounted on an $X, Y, Z$ stage that makes it possible both to focus and to choose the observation field. A heating wire (a 2-mm-long segment of a Constantan wire of $0.1 \mathrm{~mm}$ in diameter) can be placed as close as a few tens of micrometers to the film with the help of a uniaxial stage (see Ref. [9] for experimental details). Sending a pulse of very short duration then makes it possible to nucleate an edge dislocation loop [12]. Pictures are digitized on a Macintosh computer equipped with a frame grabber card. A FORTRAN routine detects the experimental shape of the loop, which is then fitted to the theoretical profile. The film thickness is calculated from the value of its reflectivity in monochromatic light [7]. The intensity of the reflected light is measured as follows: A second beam splitter casts part of the reflected light on a photodiode. A red LED connected to a function generator is used as a light source, and the photodiode signal is analyzed with the help of a lock-in amplifier (thus excluding the surrounding light). This measurement is unambiguous if the number $N$ of smectic layers is less than 25 , which is the case in all our experiments.

The evolution of the line tension $T$ of an elementary dis-

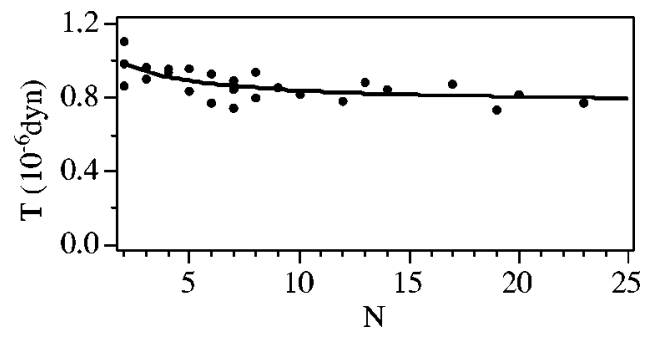

FIG. 3. Line tension as a function of the film thickness $N$.

location (of Burgers vector $b=d$ where $d$ is the layer thickness) is given in Fig. 3 as a function of the film thickness $N=D / d$ : It typically increases by $20 \%$ when the film thickness decreases from 25 to 2 layers. This increase results from the dislocation interaction with the two free surfaces as shown theoretically by Lejcek and Oswald [5]. An exact calculation leads to the following formula, provided the dislocation lies in the midplane:

$$
T=T_{\infty}+\frac{B \lambda b^{2}}{4 \sqrt{\pi \lambda d(N+1 / 2)}} \operatorname{Li}_{1 / 2}(A),
$$

where $\mathrm{Li}_{n}(x)=\sum_{p=1}^{\infty} x^{p} p^{-n}$ denotes the polylogarithm function. The parameters are $\lambda=\sqrt{K / B}$ and $A=(\gamma-\sqrt{K B}) /(\gamma$ $+\sqrt{K B}) . T_{\infty}$ denotes the line tension of an edge dislocation in an infinite medium. The other terms, proportional to $1 / \sqrt{N+\frac{1}{2}}$, represent the interaction energy with the free surfaces. Interpolation of the experimental data with $T_{\infty}$ $+\beta / \sqrt{N+\frac{1}{2}}$ leads to $T_{\infty}=(7.5 \pm 0.4) \times 10^{-7}$ dyn and $\beta$ $=(4.7 \pm 1.0) \times 10^{-7}$ dyn. The theoretical value of $\beta$ can be estimated by taking $\gamma=28.47 \mathrm{erg} / \mathrm{cm}^{2}$ [13], $d=31.6 \AA$ [14], $B \approx 5 \times 10^{7} \mathrm{erg} / \mathrm{cm}^{3}[15,16]$, and $\lambda \approx 20 \AA$ [16]. One then finds $\beta=4.2 \times 10^{-7}$ dyn, which is in very good agreement with the experimental value.

We now consider dislocations of large Burgers vectors $b=n d$. They are produced by nucleating $n$ elementary loops fitting into each other where $n$ is an integer chosen in advance. These dislocations are then grouped together with the help of the heating wire until a single dislocation of large Burgers vector is formed. We observed that the dislocations do not spontaneously assemble in films and that the thinner the film is, the more difficult this process is. For this reason, we performed this experiment in "thick" films in which surface effects are negligible (the $b^{2}$ term in the interaction

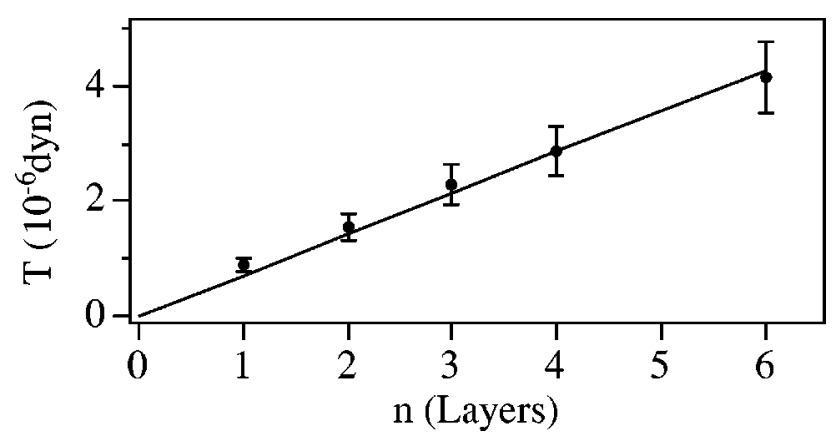

FIG. 4. Line tension of edge dislocations as a function of their Burgers vector $b=n d$ in "thick" films. 
energy obviously favors elementary edge dislocations). On the other hand, a dislocation of large Burgers vector never spontaneously dissociates when it is formed. These observations are in qualitative agreement with the theory according to which $T_{\infty}$ is proportional to $b$. This result can be obtained by assuming that the core energy is proportional to its size $r_{c}$ and to some cutoff energy $\gamma_{c}$. Minimizing $T_{\infty}$ with respect to $r_{c}$ yields [17]

$$
T_{\infty}=2 \sqrt{B \lambda \gamma_{c}} b
$$

To test this prediction quantitatively, we measured the line tension of the dislocations as a function of their Burgers vectors $b=n d$. Within experimental errors, we find that the line tension scales like $b$ in agreement with the theory (Fig. 4) and we calculate $\gamma_{c}=0.14 \mathrm{erg} / \mathrm{cm}^{2}$. These measurements suggest that the core is unchanged up to $n=6$, and does not split into two disclinations of ranks $1 / 2$ and $-1 / 2$. Williams and Kléman have first proposed that the core should split for large enough Burgers vector $[3,18,19]$. However, $b$ is in this case too small for the splitting to occur; we cannot exclude this possibility for larger $b$.

In conclusion, we have developed a very simple technique, analogous to the standard sessile drop method, for measuring the line tension of a dislocation in a vertical smectic- $A$ film. We have shown that surface effects are present in thin films and cause an increase of the line tension of the dislocations when the thickness decreases. We have also experimentally verified that the line tension of the dislocation is proportional to its Burgers vector. All these results are in very good agreement with the theoretical predictions of the elasticity theory.

This work was supported by the European Research Network under Contract No. FMRX-CT96-0085.
[1] G. Friedel, Ann. Phys. (Paris) 18, 273 (1922).

[2] F. C. Frank, Discuss. Faraday Soc. 25, 1 (1958).

[3] M. Kléman, Rep. Prog. Phys. 52, 555 (1989).

[4] J. Friedel, Dislocations (Pergamon Press, New York, 1967).

[5] L. Lejcek and P. Oswald, J. Phys. II 1, 931 (1991).

[6] C. Furtlehner and X. Leoncini (unpublished).

[7] P. Pierański et al., Physica A 194, 364 (1993).

[8] C. Y. Young et al., Phys. Rev. Lett. 40, 773 (1978).

[9] J. C. Géminard, R. Holyst, and P. Oswald, Phys. Rev. Lett. 78, 1924 (1997).

[10] A. W. Adamson, Physical Chemistry of Surfaces, 5th ed. (John Wiley \& Sons, New York, 1990).

[11] W. H. Press, B. P. Flannery, S. A. Teukolsky, and W. T. Vet- terling, Numerical Recipes (Cambridge University Press, Cambridge, 1986).

[12] The microscopic origin of this process is currently under investigation by F. Picano, J.-C. Géminard, and P. Oswald.

[13] M. Eberhard and R. B. Meyer, Rev. Sci. Instrum. 8, 67 (1996).

[14] A. J. Leadbetter et al., J. Phys. (France) 40, 375 (1979).

[15] M. Benzekri, T. Claverie, J.-P. Marcerou, and J.-C. Rouillon, Phys. Rev. Lett. 68, 2480 (1992).

[16] P. Oswald, thèse d'état, Université Paris-Sud, 1985.

[17] R. Holyst and P. Oswald, Int. J. Mod. Phys. B 9, 1515 (1995).

[18] C. E. Williams and M. Kléman, J. Phys. (France) Lett. 35, L33 (1974).

[19] P.-G. de Gennes and J. Prost, Physics of Liquid Crystals (Clarendon Press, Oxford, 1993). 